\title{
2.5D scattering of incident plane SH waves by a canyon in layered half-space
}

\author{
Zhenning $\mathrm{Ba}^{\star}$ and Jianwen Liang \\ Department of Civil Engineering, Tianjin University, Tianjin 300072, China
}

\begin{abstract}
This paper presents 2.5D scattering of incident plane SH waves by a canyon in layered half-space by the indirect boundary element method (IBEM). The free field response is carried out to give the displacements and stresses on the line which forms boundary of the canyon. The fictitious uniform moving loads are applied to the same line to calculate the Green's functions for the displacements and stresses. The amplitudes of the loads are determined by the boundary conditions. The displacements due to the free field and from the fictitious uniform moving loads have to be added to obtain the whole motion. The numerical results are carried out for the cases of a canyon in homogenous and in one layer over bedrock. The results show that the $2.5 \mathrm{D}$ wave scattering problem is essentially different from the $2 \mathrm{D}$ case, and there exist distinct differences between the wave amplification by a canyon in layered half-space and that in homogeneous half-space. The reasons for the distinct difference are explored, and the effects of the thickness and stiffness of the layer on the amplification are discussed.
\end{abstract}

Key words: canyon; layered half-space; plane SH wave; amplification; 2.5D scattering

CLC number: P315. $3^{+} 1$ Document code: A

\section{Introduction}

The effect of canyon on seismic wave propagation is a fundamental subject in seismology and earthquake engineering. The problem can be studied by analytical methods (Trifunac, 1973; Wong and Trifunac, 1974; Liang et al, 2002; Yuan and Liao, 1995) or numerical methods (Vogt et al, 1988; Wong, 1982; Sanchez-Sesma and Campillo, 1991; Kawase, 1988; Liao, 2002). Analytical methods are essential for exploring the physical nature of particular problem; however, numerical methods have advantages in solving problems with complex boundary conditions.

It should be noted that the above studies (Trifunac, 1973; Wong and Trifunac, 1974; Liang et al, 2002; Yuan and Liao, 1995; Vogt et al, 1988; Wong, 1982; Sanchez-Sesma and Campillo, 1991; Kawase, 1988; Liao, 2002) are limited to 2D problems. While for more general cases, the direction of incident wave has an angle with the axis of the canyon, and it is actually a 3D scattering problem of $2 \mathrm{D}$ structure, and it is often called 2.5D problem. Luco et al (1990) derived dynamic

\footnotetext{
* Received 23 November 2009; accepted in revised form 4 January 2010; published 10 February 2010.

* Corresponding author. e-mail: bazhenning@tju.edu.cn

(C) The Seismological Society of China and Springer-Verlag Berlin Heidelberg 2010
}

Green's functions of point force embedded in layered half-space and studied 2.5D scattering problems of canyon in a layered half-space using the wave source method. Zhang and Chopra (1991) and Stamos and Beskos (1996) used the direct boundary element method to study $2.5 \mathrm{D}$ scattering problems by canyon in homogeneous half-space. Pedersen et al (1994) presented the indirect boundary element method using full-space Green's functions to simulate 2.5D scattering problem by canyon structures. Papageorgiou and Pei (1998) also studied 2.5D problems.

In this paper, the indirect boundary element method is used to study the 2.5D scattering of a canyon in layered half-space for incident plane SH waves based on the exact dynamic stiffness of layered site (Liang and $\mathrm{Ba}$, 2007) and the dynamic Green's functions of moving load acting on an inclined line (Ba, 2008). The accuracy of the method is verified by comparison with related solutions, and numerical results are carried out for a canyon in layered half-space, and the effects of the stiffness and thickness of the layer on the amplification are discussed.

\section{Method}

For a $2.5 \mathrm{D}$ canyon problem, the wave fields at two different cross sections perpendicular to the canyon axis 
are identical but shifted with time, therefore, the wave field can be represented by moving loads acting on an inclined line (the moving velocity is equal to the apparent velocity), and we can use only one cross section of the canyon for calculation. The dynamic response by the moving loads is called 2.5D Green's functions or Green's functions of moving loads.

The model is an infinite long, uniform canyon of arbitrary cross section in a horizontally layered halfspace (Figure 1). Figure 1a is a top view of free field in plane $x-y$, and the projection on the horizontal plane normal to the wavefront forms the angle $\theta_{\mathrm{h}}$ with the axis of the canyon. Figure $1 \mathrm{~b}$ is the cross section of free field in plane $y^{\prime}-z$, and the normal to the wavefront forms the angle $\theta_{\mathrm{v}}$ with the vertical axis. Figure $1 \mathrm{c}$ is the cross section of the canyon embedded in layered half-space formed by $n$ soil layers and the bedrock.

We use the IBEM to determine the wave scattering around a canyon in a horizontally layered half-space (Figure 1). We begin with the calculation of the free field response, which can determine the three-dimensional response at the surface of the half-space and of the canyon. Then moving distributed fictitious loads are applied to the surface of the canyon discretized by lines, and the dynamic responses at the surface of the half-space and
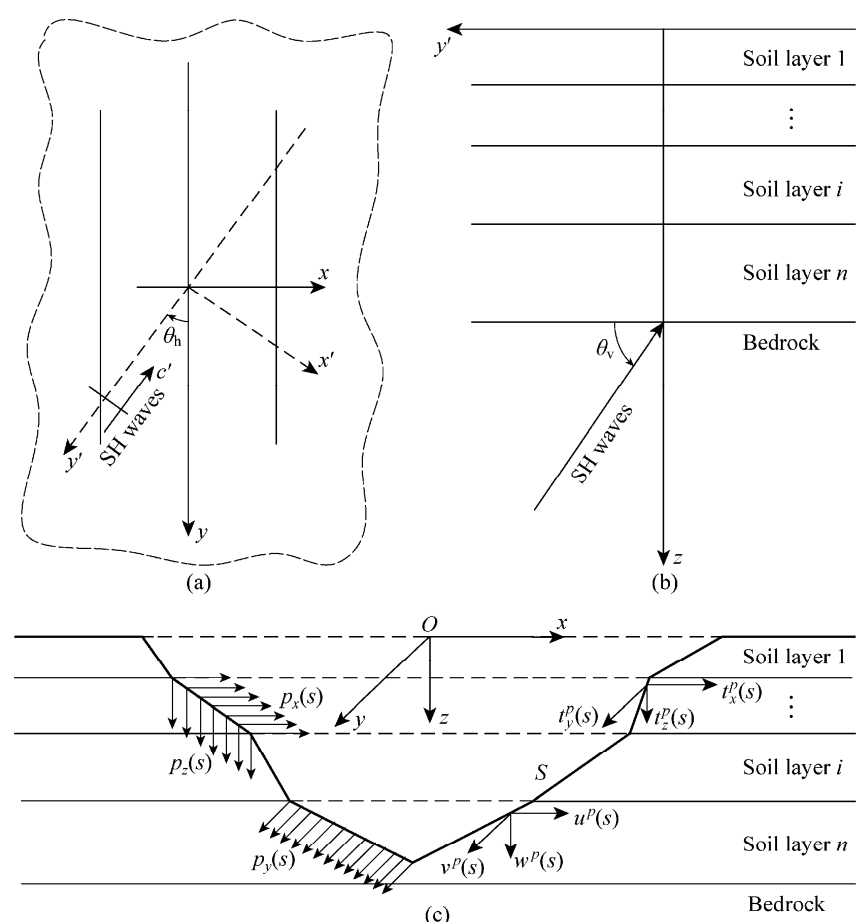

(c) Bedrock

Figure 1 The model. (a) Top view of free field; (b) cross section of free field; (c) cross section of the model. of the canyon are determined. The fictitious loads densities are determined by boundary conditions. Finally we obtain the solution by adding the responses of the free field and the responses of the fictitious loads.

\subsection{Free field response}

The apparent velocity along $x$-axis is $c_{\mathrm{S}}^{\mathrm{R}} /\left(\sin \theta_{\mathrm{h}} \cos \theta_{\mathrm{v}}\right)$, along $y$-axis $c_{\mathrm{S}}^{\mathrm{R}} /\left(\cos \theta_{\mathrm{h}} \sin \theta_{\mathrm{v}}\right)$ and along $z$ axis $c_{\mathrm{S}}^{\mathrm{R}} / \sin \theta_{\mathrm{v}}$ from Figures $1 \mathrm{a}$ and $1 \mathrm{~b}$, and the apparent velocities along $x, y$ and $z$ are $c_{\mathrm{S}}^{\mathrm{R}} / m_{x}, c_{\mathrm{S}}^{\mathrm{R}} / m_{y}$, and $c_{\mathrm{S}}^{\mathrm{R}} / m_{z}$, respectively. Then $m_{x}=\sin \theta_{\mathrm{h}} \cos \theta_{\mathrm{v}}, m_{y}=\cos \theta_{\mathrm{h}} \cos \theta_{\mathrm{v}}$ and $m_{z}=\sin \theta_{\mathrm{v}}$ where $c_{\mathrm{S}}^{\mathrm{R}}$ is the $\mathrm{S}$-waves velocity and the terms $m_{x}, m_{y}$ and $m_{z}$ are the direct cosines of incidence $\mathrm{SH}$ waves. Once $m_{x}, m_{y}$ and $m_{z}$ are obtained, the amplitudes $B_{\mathrm{P}}^{\mathrm{R}}$, $B_{\mathrm{SV}}^{\mathrm{R}}$ and $B_{\mathrm{SH}}^{\mathrm{R}}$ of the reflected waves can be expressed by the amplitudes $A_{\mathrm{P}}^{\mathrm{R}}, A_{\mathrm{SV}}^{\mathrm{R}}$ and $A_{\mathrm{SH}}^{\mathrm{R}}$ of the incident waves by introducing the zero-stress conditions of the free surface of the layered soil from equation (10) in Liang and $\mathrm{Ba}$ (2007), and the relations between $u_{0}, v_{0}, w_{0}$ and $A_{\mathrm{P}}^{\mathrm{R}}$, $A_{\mathrm{SV}}^{\mathrm{R}}, A_{\mathrm{SH}}^{\mathrm{R}}$ are established. For incident $\mathrm{SH}$ waves, $A_{\mathrm{SH}}^{\mathrm{R}}=1$ and $A_{\mathrm{P}}^{\mathrm{R}}=A_{\mathrm{SV}}^{\mathrm{R}}=0$ is introduced. For a layered half-space, if we take the bedrock outcrop as the control point and let $u_{0}, v_{0}, w_{0}$ as the input motion, the last three elements in the vector $\boldsymbol{Q}$ can be obtained by equation (1), while the other elements of the vector $\boldsymbol{Q}$ are zero, and the displacements of each layer can be solved using the direct stiffness method from equation (2).

$$
\begin{gathered}
{\left[\begin{array}{c}
P_{x 0} \\
P_{y 0} \\
\mathrm{i} P_{z 0}
\end{array}\right]=\boldsymbol{S}_{\mathrm{P}-\mathrm{SV}-\mathrm{SH}}^{\mathrm{R}}\left[\begin{array}{c}
u_{0} \\
v_{0} \\
\mathrm{i} w_{0}
\end{array}\right]} \\
\boldsymbol{S}_{\mathrm{P}-\mathrm{SV}-\mathrm{SH}} \boldsymbol{U}=\boldsymbol{Q}
\end{gathered}
$$

From equation (2) the displacements at each layer's upper and bottom interfaces can be determined, and then the amplitude coefficients of the up-going waves and down-going waves can be obtained. Finally we get the displacements and stresses at any point in the layered half-space.

In order to facilitate the solution, the stresses at the inclined line element are decomposed into the three coordinate directions as follows:

$$
\begin{aligned}
& t_{x}^{\mathrm{f}}=n_{x} \sigma_{x}^{\mathrm{f}}+n_{y} \tau_{x y}^{\mathrm{f}}+n_{z} \tau_{x z}^{\mathrm{f}}, \\
& t_{y}^{\mathrm{f}}=n_{x} \tau_{y x}^{\mathrm{f}}+n_{y} \sigma_{y}^{\mathrm{f}}+n_{z} \tau_{y z}^{\mathrm{f}},
\end{aligned}
$$




$$
t_{z}^{\mathrm{f}}=n_{x} \tau_{z x}^{\mathrm{f}}+n_{y} \tau_{z y}^{\mathrm{f}}+n_{z} \sigma_{z}^{\mathrm{f}},
$$

where $t_{x}^{\mathrm{f}}, t_{y}^{\mathrm{f}}$ and $t_{z}^{\mathrm{f}}$ are stresses along the three coordinate directions, $\sigma_{x}^{\mathrm{f}}, \sigma_{y}^{\mathrm{f}}, \sigma_{z}^{\mathrm{f}}, \tau_{x y}^{\mathrm{f}}, \tau_{x z}^{\mathrm{f}}$ and $\tau_{y z}^{\mathrm{f}}$ are the stress tensors, with the superscript " $\mathrm{f}$ " indicating the variables in the free field response. $\left(n_{x}, n_{y}, n_{z}\right)$ is the normal direction of the inclined line element and $n_{y}=0$ if the inclined line in the $x-z$ plane.

\subsection{Dynamic Green's functions}

The dynamic Green's functions of moving loads acting on inclined lines in a half-space can be solved in the following way. Firstly, the 3D dynamic Green's functions of distributed loads acting on inclined lines in a layered half-space is deduced, and then by integrating the Green's functions along the moving direction (the moving direction is the $y$-axis and the moving velocity is $c_{\mathrm{S}}^{\mathrm{R}} /\left(\cos \theta_{\mathrm{h}} \cos \theta_{\mathrm{v}}\right)$ from Figure 1), the dynamic Green's functions of moving loads acting on an inclined line are obtained. More details can be found in Ba (2008).

Let $\boldsymbol{g}_{\mathrm{u}}(x)$ and $\boldsymbol{g}_{\mathrm{s}}(x)$ be Green's functions of displacements and stresses due to moving loads, the displacements and stresses at any point in the elastic layered half-space can be expressed as

$$
\begin{gathered}
\left\{\begin{array}{c}
u^{p} \\
v^{p} \\
w^{p}
\end{array}\right\}=\boldsymbol{g}_{\mathrm{u}}(x, y, z)\left\{\begin{array}{l}
p_{x} \\
p_{y} \\
p_{z}
\end{array}\right\}, \\
{\left[\begin{array}{ccc}
\sigma_{x}^{p} & \tau_{x y}^{p} & \tau_{x z}^{p} \\
\tau_{y x}^{p} & \sigma_{y}^{p} & \tau_{y z}^{p} \\
\tau_{z x}^{p} & \tau_{z y}^{p} & \sigma_{z}^{p}
\end{array}\right]=\boldsymbol{g}_{\mathrm{s}}(x, y, z)\left\{\begin{array}{c}
p_{x} \\
p_{y} \\
p_{z}
\end{array}\right\},}
\end{gathered}
$$

where $u^{p}, v^{p}, w^{p}$ and $\sigma_{x}^{p}, \sigma_{y}^{p}, \sigma_{z}^{p}, \tau_{x y}^{p}, \tau_{z x}^{p}, \tau_{z y}^{p}$ are the displacements and stress tensors, arising form the moving loads, and $p_{x}, p_{y}$ and $p_{z}$ are densities of the moving loads.

Also in order to facilitate solution, the stresses at the inclined line element are decomposed into $x, y$ and $z$ directions as

$$
\begin{aligned}
& t_{x}^{p}=n_{x} \sigma_{x}^{p}+n_{y} \tau_{x y}^{p}+n_{z} \tau_{x z}^{p}, \\
& t_{y}^{p}=n_{x} \tau_{y x}^{p}+n_{y} \sigma_{y}^{p}+n_{z} \tau_{y z}^{p},
\end{aligned}
$$

and

$$
t_{z}^{p}=n_{x} \tau_{z x}^{p}+n_{y} \tau_{z y}^{p}+n_{z} \sigma_{z}^{p} .
$$

Substituting equation (5) into (6) gives

$$
\left\{\begin{array}{l}
t_{x}^{p} \\
t_{y}^{p} \\
t_{z}^{p}
\end{array}\right\}=\boldsymbol{g}_{\mathrm{t}}(x, y, z)\left\{\begin{array}{l}
p_{x} \\
p_{y} \\
p_{z}
\end{array}\right\}
$$

where $t_{x}^{p}, t_{y}^{p}$ and $t_{z}^{p}$ are stresses along $x, y$ and $z$ directions, $\boldsymbol{g}_{\mathrm{t}}(x, y, z)$ is the transformed Green's functions of stresses.

\subsection{Boundary conditions}

The boundary condition of the canyon can be expressed as

$$
\int_{s} \boldsymbol{W}(s)^{\mathrm{T}}\left(\left[\begin{array}{c}
t_{x}^{p}(s) \\
t_{y}^{p}(s) \\
t_{z}^{p}(s)
\end{array}\right]+\left[\begin{array}{c}
t_{x}^{\mathrm{f}}(s) \\
t_{y}^{\mathrm{f}}(s) \\
t_{z}^{\mathrm{f}}(s)
\end{array}\right]\right) \mathrm{d} s=0,
$$

where $\boldsymbol{W}(s)$ is the weighting function, which can be taken as a unit matrix, so the integral can be evaluated over each element separately. Substituting equations (3) and (7) into (8) gives

$$
\boldsymbol{T}_{p}\left\{\begin{array}{c}
p_{x} \\
p_{y} \\
p_{z}
\end{array}\right\}=\boldsymbol{T}_{\mathrm{f}}
$$

with

$$
\begin{gathered}
\boldsymbol{T}_{p}=\int_{s}[W(s)]^{\mathrm{T}} \boldsymbol{g}_{\mathrm{t}}(s) \mathrm{d} s, \\
\boldsymbol{T}_{\mathrm{f}}=-\int_{s}[W(s)]^{\mathrm{T}}\left[\begin{array}{c}
t_{x}^{\mathrm{f}}(s) \\
t_{y}^{\mathrm{f}}(f) \\
t_{z}^{\mathrm{f}}(f)
\end{array}\right] \mathrm{d} s .
\end{gathered}
$$

Combining the equations (9) and (4), the displacements in any point of the layered half-space can be expressed as

$$
\left\{\begin{array}{l}
u(x, y, z) \\
v(x, y, z) \\
w(x, y, z)
\end{array}\right\}=\left\{\begin{array}{c}
u^{\mathrm{f}}(x, y, z) \\
v^{\mathrm{f}}(x, y, z) \\
w^{\mathrm{f}}(x, y, z)
\end{array}\right\}+\boldsymbol{g}(x, y, z) \boldsymbol{T}_{p}^{-1} T_{\mathrm{f}}
$$

where $u^{\mathrm{f}}, v^{\mathrm{f}}$, and $w^{\mathrm{f}}$ are displacements along the three coordinate directions.

\subsection{Verification}

To verify our results, we compare the present results with the 2.5D results (Luco et al, 1990) in a homogenous half-space. The parameters are as follows. Poisson's ratio $v=1 / 3$, damping ratio $\zeta=0.01$, dimensionless incident frequency $\eta=2 a / \lambda_{\beta}=0.5,1.0$ and 2.0, respectively and the incident angle $\theta_{\mathrm{h}}=\theta_{\mathrm{v}}=45^{\circ}$. Figure 2 shows that the present results agree very well with those from Luco et al (1990). 

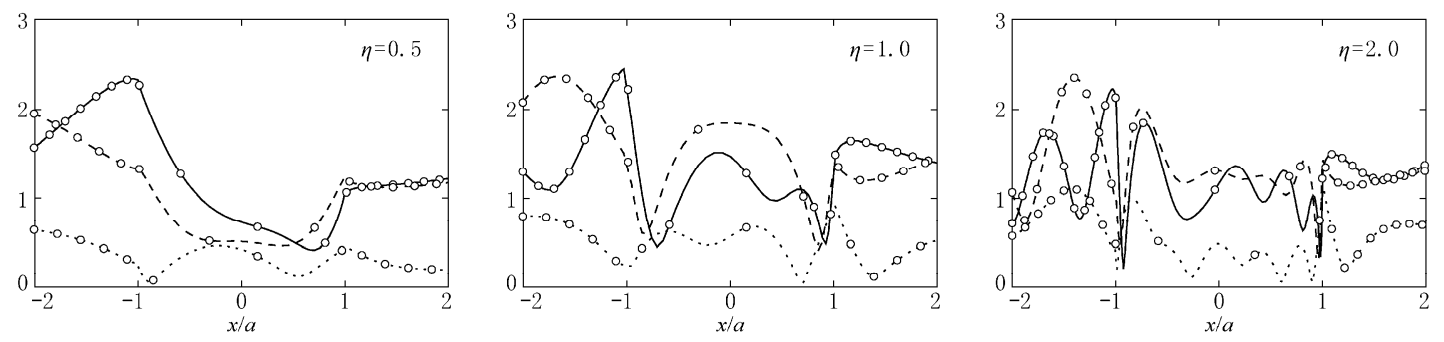

- Luco et al (1990) - $\left|u / A_{\mathrm{SH}}\right| \quad \ldots-\left|v / A_{\mathrm{SH}}\right| \quad \ldots \ldots\left|w / A_{\mathrm{SH}}\right|$

Figure 2 Comparison with 2.5D results in the case of $\theta_{\mathrm{h}}=45^{\circ}, \theta_{\mathrm{v}}=45^{\circ}, v=1 / 3$ and $\zeta=0.01$.

\section{Numerical results and discussion}

At first, we study a semi-circular canyon in a homogenous half-space, and surface displacements around the canyon are shown for different incident angles in Figure 3. The parameters are as follows. Poisson's ratio $v=1 / 3$, damping ratio $\zeta=0.001$, dimensionless frequency
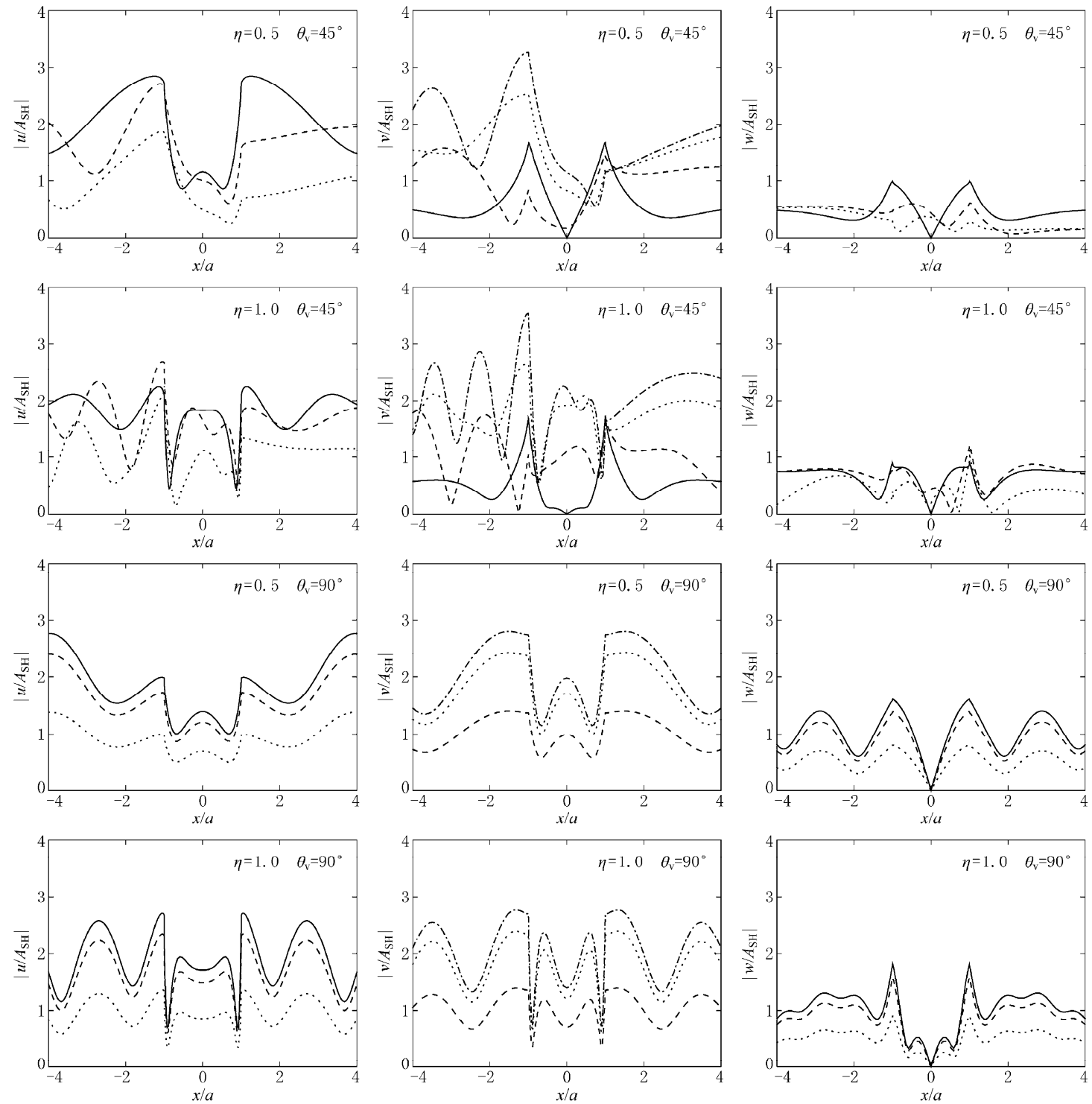

$-\theta_{\mathrm{h}}=0^{\circ}$

$--\theta_{\mathrm{h}}=30^{\circ}$

$\cdots \cdot \theta_{\mathrm{h}}=60^{\circ}$

$-\cdot \theta_{\mathrm{h}}=90^{\circ}$

Figure 3 Surface displacements around a canyon in homogenous half-space for different incident horizontal angles. 
$\eta=2 a / \lambda_{\beta}$ with $\eta=0.5$ and 1.0 , the vertical incident angle $\theta_{\mathrm{v}}=45^{\circ}$ and $90^{\circ}$, horizontal incident angle $\theta_{\mathrm{h}}=0^{\circ}, 30^{\circ}$, $60^{\circ}$ and $90^{\circ} . u, v$ and $w$ are the displacements in $x, y$ and $z$ directions, respectively; $A_{\mathrm{SH}}$ is the amplitude of the incident $\mathrm{SH}$ waves.

It is shown that the horizontal incident angle has an important effect on the amplitudes of the surface displacements. When the incident waves are perpendicular to the axis of the canyon $\left(\theta_{\mathrm{h}}=90^{\circ}\right)$, there are only the out-of-plane displacements (along $y$ direction) and the in-plane displacements (along $x$ and $z$ directions) do not exist, and the horizontal and vertical displacements are symmetric about the axis of the canyon ( $y$-axis) under $\theta_{\mathrm{h}}=0^{\circ}$. The surface displacements become more complex as the incident frequency increases and the barrier effect is more significant for the two horizontal displacements than for the vertical displacement. As the horizontal incident angle $\theta_{\mathrm{h}}$ increases, the horizontal displacements along $x$-axis increase gradually, while the horizontal displacements along $y$-axis and the vertical displacements decrease gradually, which is because as the horizontal incident angle $\theta_{\mathrm{h}}$ increases, the incident direction moves to the $x$ direction gradually and the incident $\mathrm{SH}$ waves produce out-of-plane vibration. The spatial distribution of the surface displacements become independent of the horizontal incident angle $\theta_{\mathrm{h}}$ when $\theta_{\mathrm{v}}=90^{\circ}$, but the horizontal displacements along $x$-axis are dependent on $\sin \theta_{\mathrm{h}}$, and the horizontal displacements along $y$-axis are dependent on $\cos \theta_{\mathrm{h}}$.

Next, we study a semi-circular canyon in one soil layer over bedrock, in which, $C_{\mathrm{S}}^{\mathrm{R}}$ and $\rho^{\mathrm{R}}$ are the shear velocity and material density of the bedrock; $C_{\mathrm{S}}^{\mathrm{L}}$ and $\rho^{\mathrm{L}}$ are the shear velocity and material density of the soil layer; $\zeta^{\mathrm{R}}$ and $\zeta^{\mathrm{L}}$ are the damping ratio of the bedrock and of the soil layer; $v^{\mathrm{R}}$ and $v^{\mathrm{L}}$ are the Poisson's ratio of the bedrock and of the soil layer; $H$ is the thickness of the soil layer. The dimensionless frequency is defined as $\eta=2 a / \lambda^{\mathrm{L}}$, where $\lambda^{\mathrm{L}}$ is the wavelength of the soil layer. The parameters are defined as follows. The ratio of the soil layer thickness to the semi-circular canyon radius is $H / a=2.0$; the material density ratio of the bedrock to the soil layer is $\rho^{\mathrm{R}} / \rho^{\mathrm{L}}=1.0$; the damping ratios of the soil layer and of the bedrock are $\zeta^{\mathrm{L}}=0.05$ and $\zeta^{\mathrm{R}}=0.02$, respectively; both the Poisson's ratios of the soil layer and of the bedrock are $v^{\mathrm{L}}=v^{\mathrm{R}}=1 / 3$; the horizontal incident angle is $\theta_{\mathrm{h}}=45^{\circ}$; the vertical incident angles are $\theta_{\mathrm{v}}=5^{\circ}$, $30^{\circ}, 60^{\circ}$ and $90^{\circ}$; the dimensionless frequencies are $\eta=$
$0.25,0.75$ and 1.25 ; the ratios of shear wave velocity of the bedrock to the soil layer are $C_{\mathrm{S}}^{\mathrm{R}} / C_{\mathrm{S}}^{\mathrm{L}}=2.0,5.0$ and $\infty$.

For comparison, Figure 4 shows the surface displacement of the free field. The two horizontal free field displacements are relatively large when the dimensionless frequency $\eta$ are at $0.25,0.75$ and 1.25 (first three resonance frequencies), and the maximum amplitudes of the two horizontal surface displacements in $x$ and $y$ directions are 5.12 and 5.7, while the free field displacements both are 5.07, as $\eta=0.25, \theta_{\mathrm{v}}=90^{\circ}$ and the shear wave velocity ratio of the bedrock and the soil layer is $C_{\mathrm{S}}^{\mathrm{R}} / C_{\mathrm{S}}^{\mathrm{L}}=5.0$. This is because there are resonance frequencies as the $\mathrm{SH}$ waves incident vertically from the bedrock to the soil layer (Wolf, 1985).

$$
\omega_{i}=\frac{2 i-1}{2} \pi \frac{C_{\mathrm{S}}^{\mathrm{L}}}{H} \quad i=1,2,3, \cdots
$$
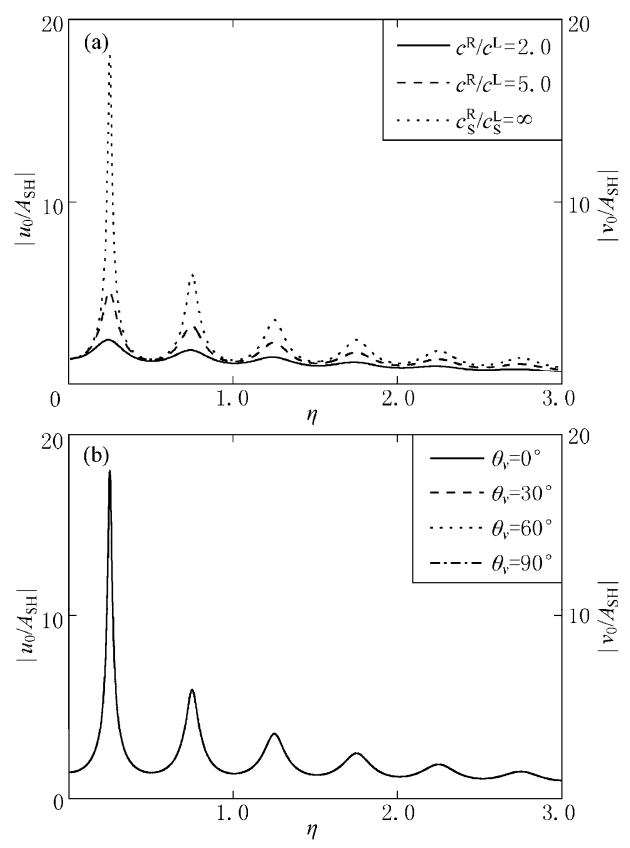

Figure 4 Surface displacements of free field for different shear wave velocity ratios of bedrock to soil layer in the case of $H / a=2.0, \theta_{\mathrm{h}}=45^{\circ}, \zeta^{\mathrm{R}}=0.02$ and $\zeta^{\mathrm{L}}=0.05$.

(a) $\theta_{\mathrm{v}}=90^{\circ}$; (b) $C_{\mathrm{S}}^{\mathrm{R}} / C_{\mathrm{S}}^{\mathrm{L}}=\infty$.

Considering the natural vibration characteristics is one of the significant features of the present model compared with the model of a canyon in a homogenous half-space.

It is shown that in Figure 5 that when the shear wave velocity ratio is relatively small $\left(C_{\mathrm{S}}^{\mathrm{R}} / C_{\mathrm{S}}^{\mathrm{L}}=2.0\right)$, the displacements are more complex in the incident side 
than those in the other side due to the barrier effect of nous half-space. It is also shown in these figures that the the canyon, which is the same as those of the homoge- surface displacements around the canyon in one soil layer
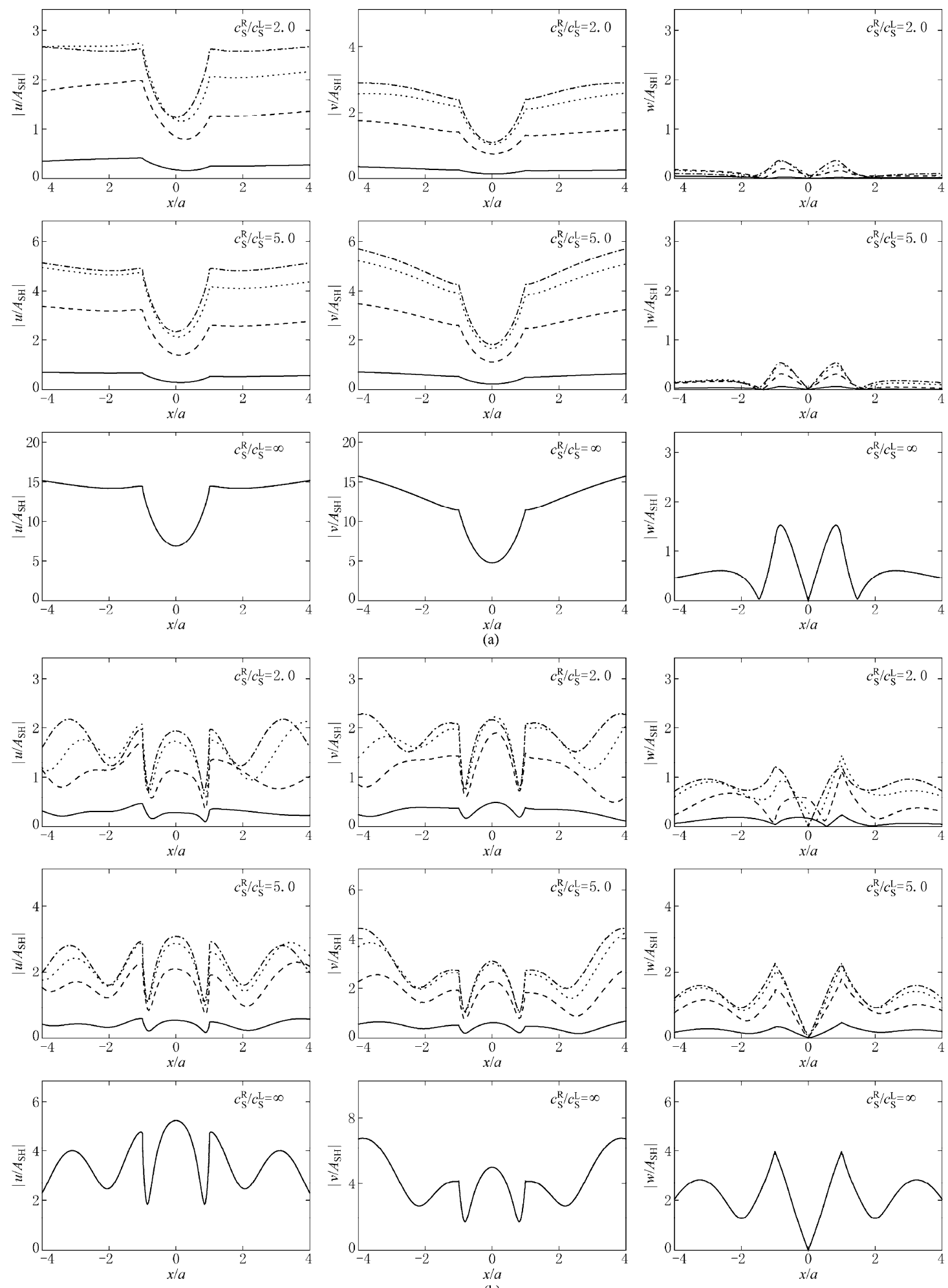

$-\theta_{\mathrm{v}}=5^{\circ} \quad-\cdots-\theta_{\mathrm{v}}=30^{\circ} \quad \ldots \cdots \theta_{\mathrm{v}}=60^{\circ} \quad-\cdots \cdot \theta_{\mathrm{v}}=90^{\circ}$

Figure 5 Surface displacements of a canyon in one soil layer over bedrock for different shear wave velocity ratios of bedrock to soil layer in the case of $H / a=2.0, \theta_{\mathrm{h}}=45^{\circ}, \zeta^{\mathrm{R}}=0.02$ and $\zeta^{\mathrm{L}}=0.05$. (a) $\eta=0.25$; (b) $\eta=0.75$; (c) $\eta=1.25$. 

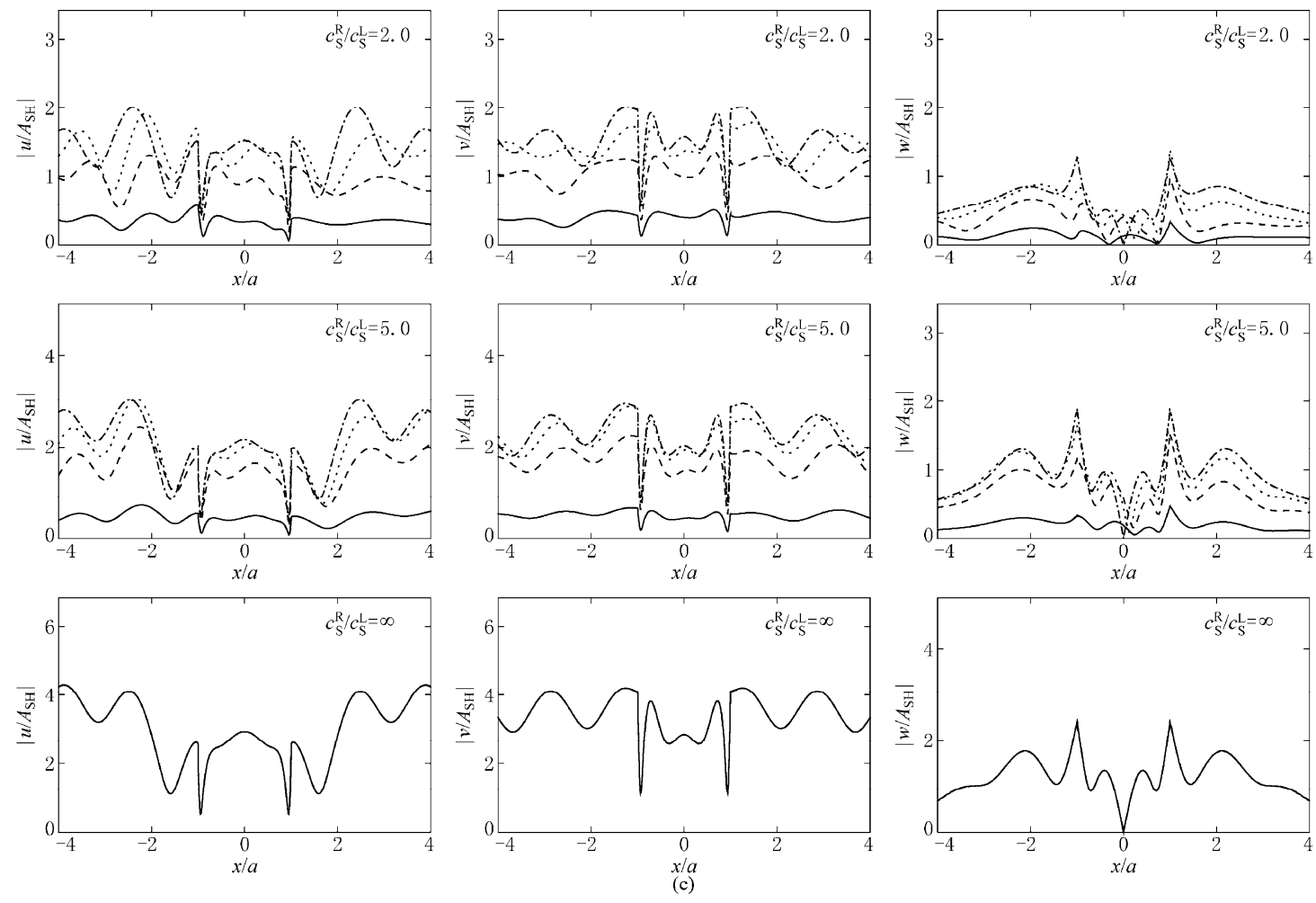

$-\theta_{\mathrm{v}}=5^{\circ}$

$--\theta_{v}=30^{\circ}$

Figure 5 Surface displacements of a canyon in one soil layer over bedrock for different shear wave velocity ratios of bedrock to soil layer in the case of $H / a=2.0, \theta_{\mathrm{h}}=45^{\circ}, \zeta^{\mathrm{R}}=0.02$ and $\zeta^{\mathrm{L}}=0.05$. (a) $\eta=0.25$; (b) $\eta=0.75$; (c) $\eta=1.25$.

over bedrock are relatively small at the vertical incident angle $\theta_{\mathrm{v}}=5^{\circ}$, which is just in contrary with those of the canyon in a homogenous half-space (the surface displacements around the canyon in a homogenous half-space are relatively large at the small vertical angle). The barrier effect becomes insignificant with the increase of the shear wave velocity ratio of the bedrock to the soil layer $\left(C_{\mathrm{S}}^{\mathrm{R}} / C_{\mathrm{S}}^{\mathrm{L}}=5.0\right)$, and the surface displacement amplitudes around the canyon become symmetry about the axis of the canyon at the non-vertical angles, and the displacements amplitude are independent of the vertical incident angle for $C_{\mathrm{S}}^{\mathrm{R}} / C_{\mathrm{S}}^{\mathrm{L}}=\infty$. This is because that the incident $\mathrm{SH}$ waves are out-of-plane waves the input motion at the bedrock only have the out-of-plane component, and in the case, if the shear wave velocity ratio of the bedrock to the soil layer become infinity, the input motion are the same in any point with no phase difference, so the input motion of the non-vertical incident angle are the same with those of the vertical incident angle (Figure $4 b$ ).

The surface displacement amplitudes become much larger as the shear wave velocity ratio of the bedrock to the soil layer increases, the reason for which is that the radiation damping of the site decreases gradually as the shear stiffness increases. For example, the maximum surface vertical displacements are 2.65, 2.76 and 2.84 at $\eta=0.25, \theta_{\mathrm{v}}=90^{\circ}$ for $C_{\mathrm{S}}^{\mathrm{R}} / C_{\mathrm{S}}^{\mathrm{L}}=2.0,5.0$ and $\infty$, respectively.

Figure 6 illustrates the surface displacements amplitudes around the canyon for different soil layer thickness $H / a=1.0$ and 4.0, respectively. The shear wave velocity ratio $C_{\mathrm{S}}^{\mathrm{R}} / C_{\mathrm{S}}^{\mathrm{L}}=5.0$, the other parameters are the same with those in Figure 5 and $\eta=0.25,0.75$ and 1.25.

Comparison of Figure 6 with Figure 5 shows that the spatial displacement distributions are very different for different soil layer thickness, which is due to the change of the resonance frequencies. For $H / a=1.0$, the vertical resonance frequencies are $\eta=0.5$ and 1.0 from equation (13), while for $H / a=4.0$, the vertical resonance frequencies are $\eta=0.125$ and 0.375 . Therefore, the dynamic characteristics of the site has important effect on the displacements around the canyon and must be paid enough attention; the spatial distribution of the surface displacements become more complex with the increase of the layer thickness for the same incident frequency; 

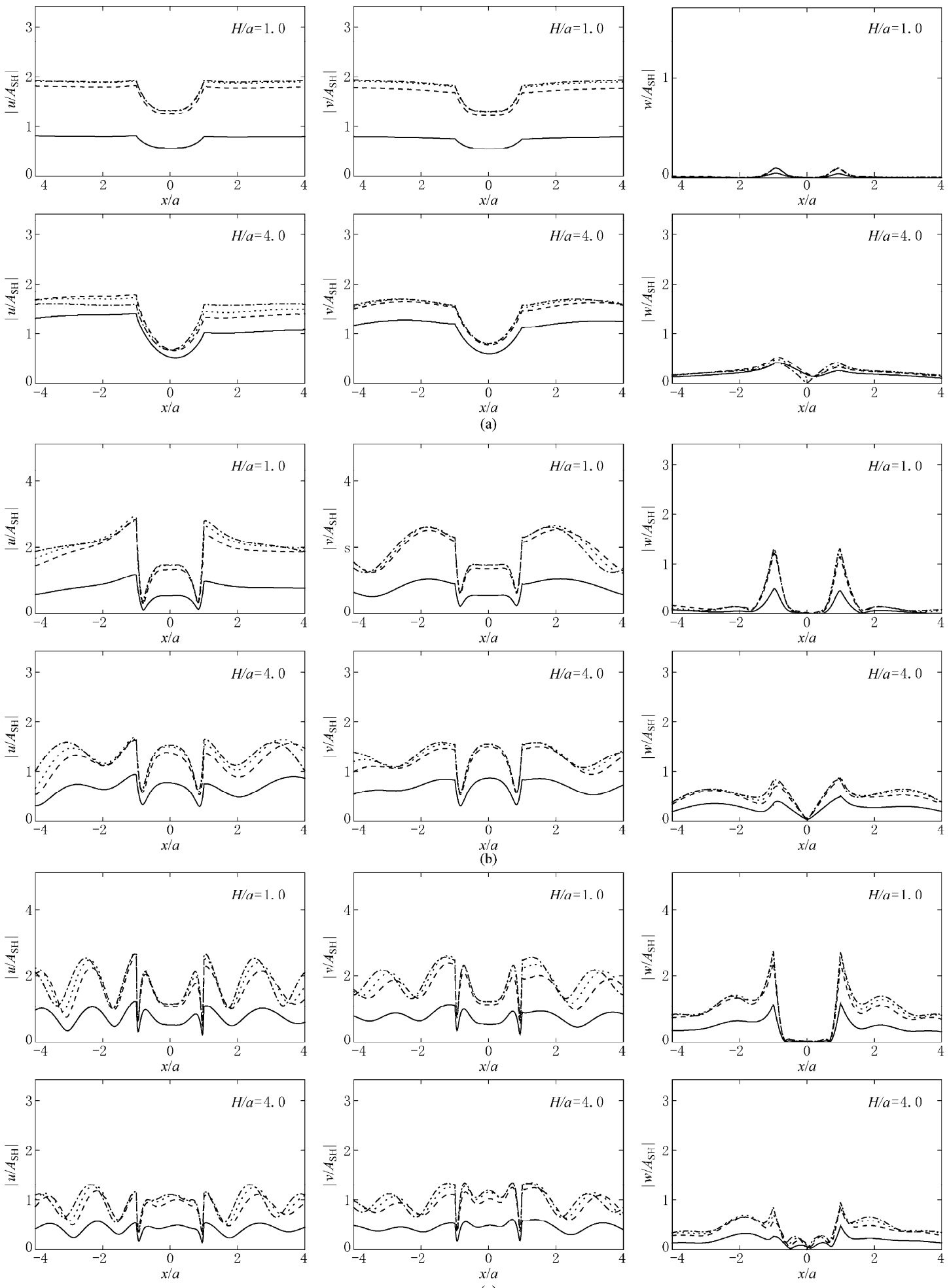

$-\theta_{\mathrm{v}}=5^{\circ}$

(c)

Figure 6 Surface displacements of a canyon in one soil layer over bedrock for different soil layer thicknesses in the case of $\theta_{\mathrm{h}}=45^{\circ}, \zeta^{\mathrm{R}}=0.02, \zeta^{\mathrm{L}}=0.05$ and $C_{\mathrm{S}}^{\mathrm{R}} / C_{\mathrm{S}}^{\mathrm{L}}=5.0$. (a) $\eta=0.25$; (b) $\eta=0.75 ;$ (c) $\eta=1.25$.

the attenuation of the surface displacements looks more obvious with the increase of the layer depth for rela- tively high incident frequency $(\eta=1.25)$. This is because we have considered the material damping and the con- 
trol point is chosen at the bedrock. The SH waves do not "feel" the soil layer and the canyon for thinner soil layer $(H / a=1.0)$ and lower incidence frequency $(\eta=0.25)$ and the same phenomenon can also be found in the two dimensional case. For this case, the surface displacements are very close to the free field surface displacements and the two horizontal surface displacements are almost the same not only in the spatial distribution but also in the amplitude.

\section{Conclusions}

The 2.5D scattering of incident plane $\mathrm{SH}$ waves by a canyon in a layered half-space is presented by using the indirect boundary element method (IBEM) and the dynamic Green's functions of moving loads acting on an inclined line in a layered half-space. The present method has two advantages. Firstly, there is no singularity due to the Green's functions of uniform loads acting on an inclined line in a layered half-space. Secondly, the presented method is particularly appropriate to elastic wave problems in a layered medium. The accuracy of the method is verified by comparison with the related solutions. The numerical results are carried out for the cases of a canyon in homogenous and in one layer over bedrock. Some conclusions are obtained.

The $2.5 \mathrm{D}$ wave scattering problem is essentially different from the 2D case, and the horizontal incident angle has an important effect on the surface displacement amplitudes. There exist distinct differences between the wave amplification by a canyon in layered half-space and that in homogeneous half-space. The dynamic characteristics of the soil layer have significant effect on both the amplitudes and frequency spectrum of the surface displacements, which must be paid enough attention.

It should be noted that this study can be used for the wave scattering of a canyon of arbitrary shape, although a semi-circular canyon in one soil layer over bedrock is studied in the paper.

Acknowledgements This study is jointly supported by National Natural Science Foundation of China (Nos. 50908156 and 50978183).

\section{References}

Ba Z (2008). Green's Functions for Layered Half-Space and Elastic Wave Scattering by Local Sites. PhD Dissertation, Tianjin University, Tianjin, 72-98 (in Chinese with English abstract).

de Barros F C P and Luco J E (1994). Response of a layered viscoelastic half-space to a moving point load. Wave Motion 19: 189-210.

Dravinski M and Mossessian T K (1987). Scattering of plane harmonic P, SV, and Rayleigh waves by dipping layers of arbitrary shape. Bull Seism Soc Amer 77: 212-235.

Kawase H (1988). Time-domain response of a semi-circular canyon for incident SV, P, and Rayleigh waves calculated by the discrete wavenumber boundary element method. Bull Seism Soc Amer 78: 1415-1 437.

Liang $\mathrm{J}$ and $\mathrm{Ba} Z$ (2007). Exact dynamic stiffness matrices of 3-D layered site and its Green's functions. Earthq Eng Eng Vib 27(5): 7-17 (in Chinese with English abstract).

Liang J, Yan L and Lee V W (2002). Scattering of incident plane P waves by a circular-arc canyon with a coving layer. Acta Mechanica Solida Sinica 23(4): 397-411.

Liao Z (2002). Introduction to Wave Motion Theories in Engineering. Second edition. Science Press, Beijing, 236-270 (in Chinese).

Luco J E, Wong H L and de Barros F C P (1990). Three dimensional response of a cylindrical canyon in a layered halfspace. Int J Earthq Eng Struct Dynam 19: 799-817.

Papageorgiou A and Pei D (1998). A discrete wave number boundary element method for study of 3-D response of 2-D scatterers. Earthq Eng Struct Dynam 27(6): 619-638.

Pedersen H, Sanchez-Sesma F J and Campillo M (1994). Three-dimensional scattering by two-dimensional topographies. Bull Seism Soc Amer 84: 1 169-1 183 .

Sanchez-Sesma F J and Campillo M (1991). Diffraction of P, SV, and Rayleigh waves by topographic features: A boundary integral formulation. Bull Seism Soc Amer 81: 2234-2 253.

Stamps A A and Beskos D E (1996). 3-D seismic response analysis of long lined tunnels in half-space. Soil Dyn Earthq Eng 15: 111-118.

Trifunac M D (1973). Scattering of plane SH wave by a semi-cylindrical canyon. Earthq Eng Struct Dynam 1: 267-281.

Vogt R F, Wolf J P and Bachmann H (1988). Wave scattering by a canyon of arbitrary shape in a layered half-space. Earthq Eng Struct Dynam 16: $803-812$.

Wolf J P (1985). Dynamic Soil-Structure Interaction. Englewood Cliffs, Prentice-Hall, 160-164.

Wong H L (1982). Effect of surface topography on the diffraction of P, SV and Rayleigh waves. Bull Seism Soc Amer 72(4): 1 167-1 183.

Wong H L and Trifunac M D (1974). Scattering of plane SH-waves by a semi-elliptical canyon. Earthq Eng Struct Dynam 3: 157-169.

Yuan X and Liao Z (1995). Scattering of plane SH waves by a cylindrical alluvial valley of circular-arc cross-section. Earthq Eng Struct Dynam 24: 1303-1313.

Zhang L and Chopra A K (1991). Three-dimensional analysis of spatially varying ground motions around a uniform canyon in a homogeneous half-space. Int J Earthq Eng Struct Dynam 20: 911-926. 